\title{
Doublex Sequencing in Molecular Diagnosis of Hereditary Diseases
}

BioTechniques 24:838-841 (May 1998)

\author{
J. Plaschke, H. Voss ${ }^{1}$, M. \\ Hahn, W. Ansorge ${ }^{1}$ and H.K. \\ Schackert \\ Technical University, Dresden, \\ and ${ }^{1}$ European Molecular Bio- \\ logy Laboratory, Heidelberg, \\ Germany
}

\section{INTRODUCTION}

In addition to numerous screening techniques for mutation detection, DNA sequencing is still the ultimate technique for highly accurate characterization of mutations on the DNA level. There is an increasing demand for highthroughput sequencing of disease-associated genes in clinical samples. Solidphase sequencing of single-stranded (ss)DNA using dye-labeled primers (2) has proven to be a highly reliable system for clinical diagnostics, where unambiguous detection of point mutations in heterozygous patient DNA is possible because of uniform signals generated by T7 DNA polymerase (1). Recently, a modified form of Taq DNA polymerase termed Thermo Sequenase $^{\mathrm{TM}}$ was introduced (7). This modified form generates uniform signals in combination with dye-labeled primers and also makes cycle sequencing attractive for clinical diagnostics. We describe the combined use of an ALF DNA Sequencer ${ }^{\mathrm{TM}}$ and an ALFexpress $^{\mathrm{TM}}$ DNA Sequencer for analysis of tumor-suppressor genes routinely sequenced in our laboratory. Sequencing reaction products were generated simultaneously from two template strands using a fluorescein isothiocyanate (FITC)- and a Cy5 $5^{\mathrm{TM}}$-labeled primer together in the same reaction. We tested doublex sequencing as follows: $(i)$ on short polymerase chain reaction (PCR) products to determine the sequence completely on both strands; (ii) on longer PCR products (800-1200 bp) to generate an overlap to determine the complete sequence from a single reaction; and (iii) on two independent PCR products (150-400 bp), comparing the performance of cycle sequencing on double-stranded (ds)DNA vs. isothermal sequencing on ssDNA.

\section{MATERIALS AND METHODS \\ DNA Samples}

Genomic DNA was extracted from peripheral blood or from normal mucosa specimens of hereditary nonpolyposis colorectal cancer (HNPCC) patients and from peripheral blood of patients with breast cancer using the QIAamp ${ }^{\circledR}$ Blood Kit and the QIAamp Tissue Kit, respectively (both from Qiagen GmbH, Hilden, Germany).

\section{PCR Amplification}

For HNPCC mutation analysis, exons of the two mismatch repair genes, $h M S H 2$ and $h M L H 1$, including their flanking intron sequences, were amplified by PCR, applying primer sequences and PCR conditions as described by Kolodner et al. $(4,5)$. One primer of each pair carried the M13 universal or the M13 reverse primer sequence at the 5 ' end. PCR products for T7 DNA polymerase sequencing were generated using $5^{\prime}$ biotinylated antisense primers to allow $\mathrm{NaOH}$-mediated strand separation after binding to streptavidin-coated magnetic beads (2). Various parts of exon 11 of the breast cancer-related gene $B R C A 1$ were amplified from genomic DNA, resulting in $0.8-1.2-\mathrm{kb}$ fragments. Primers and PCR conditions were as published (6), except that primers were modified as described above. For fragments not containing biotin, 20 $\mu \mathrm{L}$ of the PCR were separated on $1 \%$ agarose gels (SERVA, Heidelberg, Germany). Fragments were excised from the gel, eluted in $50 \mu \mathrm{L}$ sterile water at $65^{\circ} \mathrm{C}$ for $2 \mathrm{~h}$ or at $35^{\circ} \mathrm{C}$ overnight and checked for purity and concentration on agarose gels using $8 \mu \mathrm{L}$ of the eluate.

\section{Sequencing}

For simultaneous sequencing, an FITC-labeled primer was always used 
in combination with a Cy5-labeled primer (Amersham Pharmacia Biotech, Freiburg, Germany). Primers were ei- ther M13 universal, M13 reverse or gene fragment-specific, as used for PCR. Cycle sequencing was performed
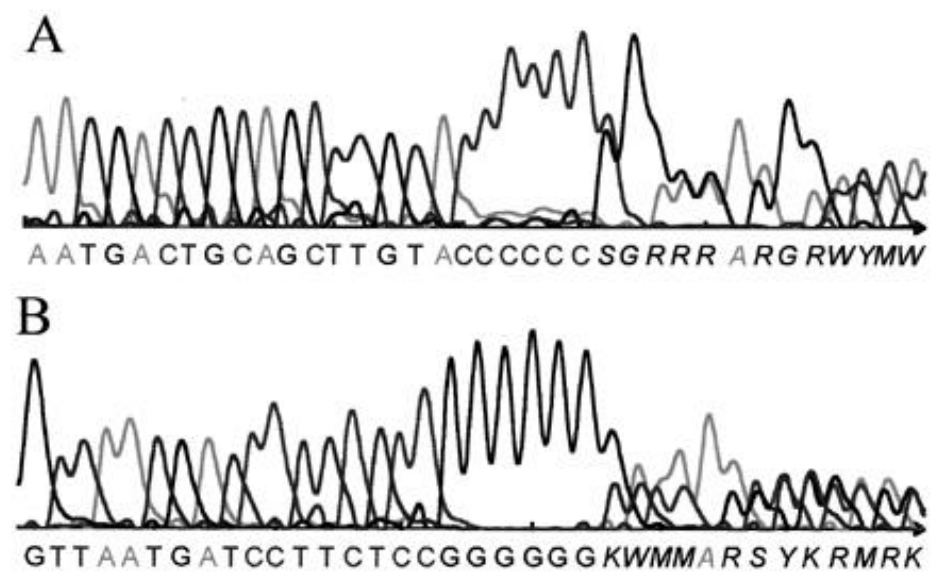

Figure 1. Exon 13 of human mismatch repair gene $h M L H 1$ cycle-sequenced bidirectionally with an FITC-labeled M13 universal primer (A) and a Cy5-labeled specific antisense primer (B) in a single reaction. Both sequences show a $\mathrm{C}$ or $\mathrm{G}$ insertion (respectively) for one allele, resulting in a frameshift.

using the Thermo Sequenase Fluorescent Labelled Primer Cycle Sequencing Kit (Amersham Pharmacia Biotech). Five microliters of the eluate and 1 pmol of each of the two labeled primers were used in combination with $2 \mu \mathrm{L}$ of the respective $\mathrm{A}, \mathrm{C}, \mathrm{G}$ and $\mathrm{T}$ reaction mixtures. Cycling conditions were: initial denaturation for $2 \mathrm{~min}$ at $94^{\circ} \mathrm{C}, 20$ times $\left(94^{\circ} \mathrm{C}\right.$ for $30 \mathrm{~s}, 60^{\circ} \mathrm{C}$ for $\left.30 \mathrm{~s}\right)$ and a final extension for $3 \mathrm{~min}$ at $72^{\circ} \mathrm{C}$. Reactions were terminated by adding $8 \mu \mathrm{L}$ of stop solution (deionized formamide containing $5 \mathrm{mg} / \mathrm{mL}$ dextran blue). Samples were heat-denatured, and half of the reaction products were loaded on an ALF DNA Sequencer (detecting FITC) while the other half were loaded on an ALFexpress DNA Sequencer (detecting Cy5) (both from Amersham Pharmacia Biotech). Electrophoresis was performed using standard conditions. For isothermal ssDNA sequencing with T7 DNA polymerase, two 


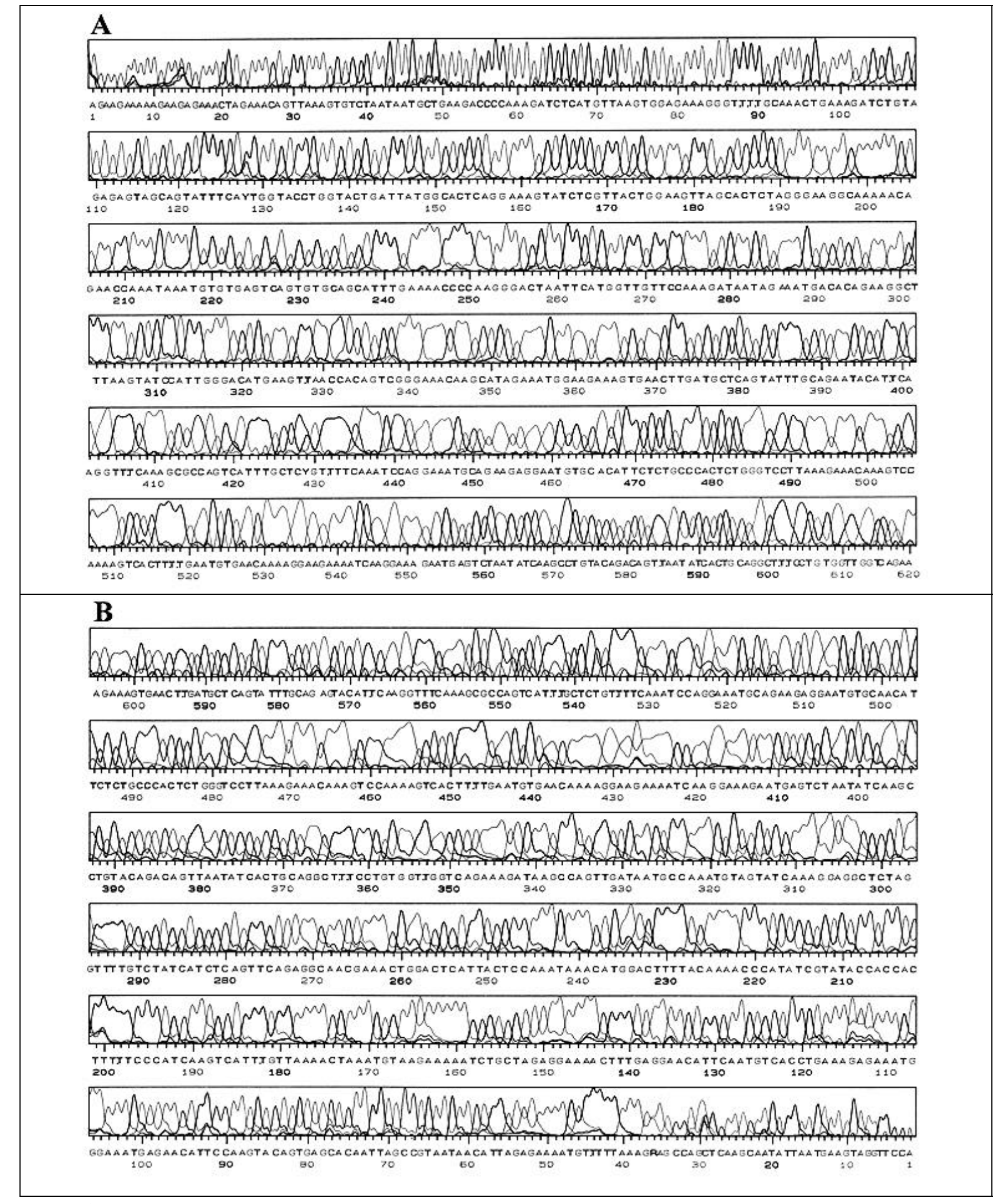

Figure 2. Sequence of BRCA1 nt 2303-3267 (exon 11) generated simultaneously using a Cy5-labeled specific sense primer (A) and an FITC-labeled M13 reverse primer (B). The sequences overlap each other for 260 bases. Heterozygous situations are detected at positions 128 and 429 of the sense strand and at position 36 of the antisense strand. 
PCR products were mixed, strand-separated using Dynabeads ${ }^{\circledR}$ M-280 (Dynal, Hamburg, Germany) and sequenced applying the AutoRead ${ }^{\mathrm{TM}}$ Sequencing Kit (Amersham Pharmacia Biotech) according to the manufacturer's protocol.

\section{RESULTS AND DISCUSSION}

Recently, Wiemann et al. (8) described the principle of doublex simultaneous sequencing of both DNA strands in the same sequencing reaction and analysis on the European Molecular Biology Laboratory (EMBL) prototype 2-dye DNA sequencing device using T7 DNA polymerase and recombinant template DNA. Kilger and Pääbo (3) analyzed simultaneous sequencing of both strands on the human mitochondrial control region by direct exponential amplification and sequencing (DEXAS). We investigated the doublex technology for sequencing of human genomic PCR products in molecular diagnosis, where the highest accuracy is necessary for unambiguous determination of point mutations. It turned out to be particularly valuable for several reasons:

(i) The technology allows even more accuracy in diagnostics, because both strands of a human genomic PCR product can be completely determined in a single sequencing reaction if the sequence to be analyzed is shorter than the resolution limit of the sequencing gel. Figure 1 shows sequences obtained from exon 13 (290-bp fragment) of the human mismatch repair gene $h M L H 1$. Both sequences show a heterozygous situation starting at the region of six Cs and Gs because of the insertion of an additional $\mathrm{C}$ or $\mathrm{G}$ in one of the two alleles. The reverse sequence confirms the wild-type sequence for this exon downstream to the insertion.

(ii) Doublex cycle sequencing on both strands allows the reading length to be increased by generating an overlap to determine the complete sequence of a larger PCR product. Fragments of the BRCA1 gene, $0.8-1.2 \mathrm{~kb}$, were sequenced simultaneously from both ends. Automated base calling was up to 800 bases for the individual sequences to yield up to 1500 bases from both sequences of a single reaction. Figure 2 shows the sequences of a fragment spanning from nucleotides (nt) 22483290 (1043 bp). Both sequences call about 600 bases and overlap each other for 260 bases. The complete sequence can be determined from high-accuracy regions to exclude failure in heterozygote detection. Three common polymorphisms located within this fragment, at positions 128 and 429 of the sense strand and at position 36 of the antisense strand, were unambiguously detected. The second polymorphism could not be determined in the antisense strand at position 538, which was identical to both FITC- and Cy5-dye sequences generated separately. Nonetheless, the Cy5 platform, with its 20fold increased dynamic range compared to the FITC platform, might more accurately detect heterozygous situations because of a higher signal-tonoise ratio.

(iii) Short genomic PCR products (150-400 bp), which most exons of tumor-suppressor genes are, are suitable for simultaneous sequencing of two fragments both by cycle sequencing of double-stranded templates with Thermo Sequenase and by isothermal T7 DNA polymerase-based sequencing after strand separation of PCR products on solid support. We investigated pooling of products on exons 6 and 10, 7 and 11 , and 8 and 13 of the mismatch repair gene $h M S H 2$ and generated unambiguous sequences for both exons and with both polymerases (data not shown). Immobilization of more than one PCR product to one magnetic bead reduces the sequencing signals by at least $50 \%$ and therefore might restrict the use of this approach for the analysis of heterozygous samples.

In the future, there will be increasing demand for molecular diagnosis. Doublex sequencing should prove to be useful because it allows a higher throughput while maintaining the same costs without the need for additional reagents, enzymes or nucleotides. A sufficient amount of product is generated to allow sample division and independent analysis on two existing commercial sequencing devices operating in the four-lane, one-dye mode and FITC and Cy5 dyes. Among the various combinations tested, we found no difference in reading length, base-calling quality or detection of heterozygotes compared to separately generated sequences. This technique may be even more attractive if the sequencing devices have several lasers available in a single instrument.

\section{REFERENCES}

1.Hopgood, R., K.M. Sullivan and P. Gill. 1992. Strategies for automated sequencing of human mitochondrial DNA directly from PCR products. BioTechniques 13:82-92.

2.Hultman, T., S. Stahl, E. Hornes and M. Uhlen. 1989. Direct solid phase sequencing of genomic and plasmid DNA using magnetic beads as solid support. Nucleic Acids Res. 17:4937.

3.Kilger, C. and S. Pääbo. 1997. Direct exponential amplification and sequencing (DEXAS) of genomic DNA. Biol. Chem. 378:99-105.

4.Kolodner, R.D., N.R. Hall, J. Lipford, M.F. Kane, P.T. Morrison, P.J. Finan, J. Burn, P. Chapman et al. 1995. Structure of the human $M L H I$ locus and analysis of a large hereditary nonpolyposis colorectal carcinoma kindred for mlh1 mutations. Cancer Res. 55:242-248.

5.Kolodner, R.D., N.R. Hall, J. Lipford, M.F. Kane, M.R.S. Rao, P. Morrison, L. Wirth, P.J. Finan et al. 1994. Structure of the human MSH2 locus and analysis of two Muir-Torre kindreds for msh2 mutations. Genomics 24:516-526.

6.Simard, J., P. Tonin, F. Durocher, K. Morgan, J. Rommens, S. Gingras, C. Samson, J.-F. Leblanc et al. 1994. Common origins of BRCA1 mutations in Canadian breast and ovarian cancer families. Nature Genet. 8:392-398.

7.Tabor, S. and C.C. Richardson. 1995. A single residue in DNA polymerases of E. coli DNA polymerase I family is critical for distinguishing between deoxy- and dideoxyribonucleotides. Proc. Natl. Acad. Sci. USA 92:6339-6343.

8.Wiemann, S., J. Stegemann, D. Grothues, A. Bosch, X. Esttivill, C. Schwager, J. Zimmermann, H. Voss and W. Ansorge. 1995. Simultaneous on-line DNA sequencing on both strands with flourescent dyes. Anal. Biochem. 224:117-121.

Received 18 July 1997; accepted 12 January 1998.

Address correspondence to:

Dr. Jens Plaschke

Department of Surgical Research

Carl Gustav Carus Klinikum

Technical University Dresden

Fetscherstr. 74, 01307 Dresden, Germany

Internet:plaschke@rcs.urz.tu-dresden.de 